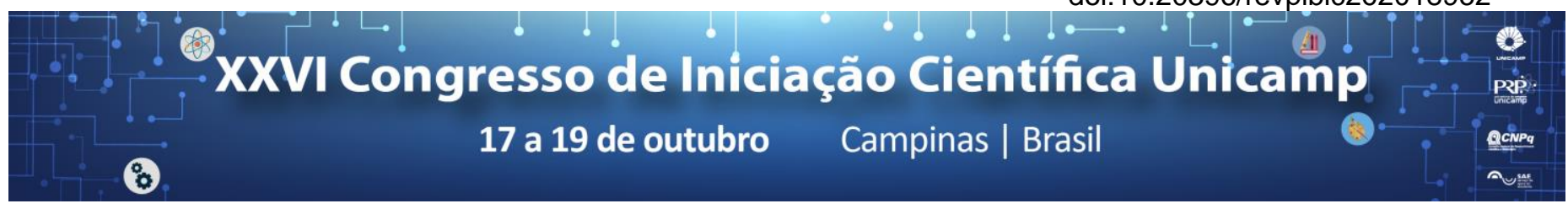

\title{
Identification of multi-crosslinked species by mass spectrometry
}

\author{
Luiz H. F. Araujo, Fabio C. Gozzo.
}

\begin{abstract}
A search for multiple crosslinking reaction products was conducted using processed raw data of the protein SALBIII obtained by LC/MS-MS. In parallel, new compounds containing three reactive terminal groups were used with myoglobin and cytochrome $\mathrm{c}$ to evaluate the formation of multiple crosslinked species.
\end{abstract}

\section{Key words:}

Mass spectrometry, Proteomics, Protein.

\section{Introduction}

The use of mass spectrometry (MS) on the analysis of cross linking reactions allows the identification of molecular species, which give extremely useful information regarding spatial distance restriction from linked residues united by a cross linking agent, using all the advantages of MS. The crosslinking technique is based on the union of two molecules by covalent bond using multifunctional organic compounds with terminal reactive groups, which are separated by a variable length space arm. When these species are put in contact with a protein, they are capable of bind covalently to the side chains of some sort of amino acids, which can vary according to their selectivity. ${ }^{1,2}$

\section{Results and Discussion}

The raw data of the digests obtained on the reaction between the protein complex SALBIII and the crosslinking agent hexamethylenediamine (HEX), with posterior tryptic digestion, was already available in the lab.

The files containing the raw data of the LC-MS/MS runs were processed on the software Mascot Distiller to obtain the precursors mass/charge ratio and, consequently, their charge. Using the $\mathrm{m} / \mathrm{z}$ information the candidate masses were calculated, with the result being expressed as monocharged species experimentally observed. In order to be considered a good candidate the mass error allowed was up to $30 \mathrm{ppm}$. The most promising specie, with an error of $5 \mathrm{ppm}$, had its MS/MS spectrum analyzed, but its fragmentation pattern was not the one expected. Therefore, even if the calculated mass to a specie was very close to a peptide experimentally observed, their MS/MS spectrum did not match.

In Chart $\mathbf{1}$ are described the digested peptides, named as $\mathrm{P} 1, \mathrm{P} 2$ and $\mathrm{P} 3$, the amino acid residues, described as $\mathrm{K}$ and $\mathrm{S}$ (lysine and serine, respectively) and their location, given by the following number. In this study the monocharged species masses were annotated, taking into account the crosslinking agent incorporation, namely disuccinimidyl suberate (DSS), and the release of four water molecules.

The second study was the test of new crosslinking agents, such as glycerol, triethanolamine and tris(2aminoethyl)amine in myoglobin and cytochrome $c$ (from horse heart). The novelty in this case is the attempt of three terminal reactive groups compounds to produce threepeptides bonded species. This step is still in progress and conditions adjustment are being made, but the results obtained in $\mathrm{pH}=6,0$ to myoglobin indicate the formation of three and four dead ends, which is less than expected. In the cytochrome $\mathrm{c}$ the crosslinked species were not observed, which is probably due to its low lysine content.

Chart 1. Possible multiple crosslinked peptides of SALBIII protein.

\begin{tabular}{cc} 
Description & $\begin{array}{c}\text { Possible } \\
\text { species }-\mathrm{P} 1+ \\
\text { P2 + P3 + (H+) } \\
+ \text { DSS - 4H } \mathbf{O}\end{array}$ \\
\hline P1/P2 (K6/K113) P2/P3 (K99/S131) & 7493,7632 \\
\hline P1/P2 (K8/K113) P2/P3 (K99/S132) & 7649,8418 \\
\hline P1/P2 (K6/K113) P2/P3 (K99/S132) & 8252,1264 \\
\cline { 2 - 2 } & 7649,8418 \\
\hline P1/P2 (K8/K113) P2/P3 (K99/K136) & 7493,7632 \\
\hline P1/P2 (K6/K113) P2/P3 (K99/K136) & 7493,7632 \\
\hline
\end{tabular}

\section{Conclusions}

The presence of species containing multiple crosslinks was not observed in the study with SALBIII using DSS as the crosslinking agent. A list of possibilities was created using the data displayed on the software SIM-XL and the precursors candidates masses were compared with the raw data obtained by the software Mascot Distiller. The last experiment was focusing in the application of new crosslinking agents to give even more spatial restriction information using myoglobin and cytochrome c.

\section{Acknowledgement}

To FAPESP (Grant 2014/17264-3), PIBIC/CNPq and Institute of Chemistry UNICAMP.

\footnotetext{
${ }^{1}$ Ozawa, K.; Wu, P. S. C.; Dixon, N. E.; Otting, G. FEBS Journal 2006, 273, 4154-4159.

2 Sinz, A. Mass Spectrom. Rev. 2006, 25, 663-82.

${ }^{3}$ Lima, D. B.; de Lima, T. B.; Balbuena, T. S.; Neves-Ferreira, A. G.; Barbosa,

V. C.; Gozzo, F. C.; Carvalho, P. C. J. Proteomics 2015, 129, 51-5
} 